Please do not remove this page

RMIT

UNIVERSITY

\title{
The inverse hexagonal - inverse ribbon - lamellar gel phase transition sequence in low hydration DOPC:DOPE phospholipid mixtures
}

Kent, Benjamin; Garvey, Chris; Cookson, David; Bryant, Gary

https://researchrepository.rmit.edu.au/esploro/outputs/9921864153001341/filesAndLinks?institution=61RMIT_INST\&index=null

Kent, B., Garvey, C., Cookson, D., \& Bryant, G. (2009). The inverse hexagonal - inverse ribbon - lamellar gel phase transition sequence in low hydration DOPC:DOPE phospholipid mixtures. Chemistry and Physics of Lipids, 157(1), 56-60. https://doi.org/10.1016/j.chemphyslip.2008.10.003

Document Version: Submitted Version

Published Version: https://doi.org/10.1016/j.chemphyslip.2008.10.003

Repository homepage: https://researchrepository.rmit.edu.au

(C) 2008 Elsevier Ireland

Downloaded On 2023/04/26 22:54:12 +1000

Please do not remove this page 
Thank you for downloading this document from the RMIT Research Repository.

The RMIT Research Repository is an open access database showcasing the research outputs of RMIT University researchers.

RMIT Research Repository: http://researchbank.rmit.edu.au/

\section{Citation:}

Kent, B, Garvey, C, Cookson, D and Bryant, G 2009, 'The inverse hexagonal - inverse ribbon - lamellar gel phase transition sequence in low hydration DOPC:DOPE phospholipid mixtures', Chemistry and Physics of Lipids, vol. 157, no. 1, pp. 56-60.

See this record in the RMIT Research Repository at:

http://researchbank.rmit.edu.au/view/rmit:4799

Version: Submitted Version

Copyright Statement: (C) 2008 Elsevier Ireland

Link to Published Version:

http://dx.doi.org/10.1016/j.chemphyslip.2008.10.003 


\title{
The inverse hexagonal - inverse ribbon - lamellar gel phase transition sequence in dehydrated DOPC:DOPE phospholipid mixtures.
}

\author{
Ben Kent,${ }^{1}$ Christopher J. Garvey, ${ }^{2}$ David Cookson, ${ }^{3}$ and Gary Bryant ${ }^{1, *}$ \\ ${ }^{1}$ Applied Physics, School of Applied Sciences, RMIT University, Melbourne, VIC 3000, Australia \\ ${ }^{2}$ Australian Nuclear Science and Technology Organisation, PMB 1, Menai, NSW 2334, Australia \\ ${ }^{3}$ Australian Synchrotron, 800 Blackburn Road, Clayton, VIC 3068, Australia
}

(Dated: April 22, 2008)

\begin{abstract}
The inverse hexagonal to inverse ribbon phase transition in a mixed phosphatidylcholinephosphatidylethanolamine system at low hydration is studied using Small and Wide Angle X-ray scattering. It is found that the structural parameters of the inverse hexagonal phase are independent of temperature. By contrast the length of each ribbon of the inverse ribbon phase increases continuously with decreasing temperature over a range of $50{ }^{\circ} \mathrm{C}$. At low temperatures the inverse ribbon phase is observed to have a transition to a gel lamellar phase, with no intermediate fluid lamellar phase. This phase transition is confirmed by Differential Scanning Calorimetry. The biological relevance of these findings to dehydration and freezing damage are discussed.
\end{abstract}

The transitions of naturally occurring phospholipids from the biologically stable bilayer phase to various nonlamellar phases, has been the subject of considerable interest for decades eg [1-7]. Such phases are known to occur in membrane systems with low water contents, and thus are particularly important in understanding dehydration and freezing damage in biological organisms eg [8-13]. In addition to their importance at low hydrations, these phases may be involved in cell fusion [7, 14-16].

The inverse hexagonal phase $\left(H_{I I}\right)$, which consists of thin channels of water surrounded by lipid head groups, arranged on a 2D hexagonal lattice, has been widely studied [1-7]. An equally important phase which has received less attention is the inverse ribbon phase $\left(P_{\alpha}\right)$, which consists of ribbon-like strips of bilayer packed on a two-dimensional rectangular lattice. A schematic of both phases is shown in Fig. 1. The importance of such phases in biology is that they disrupt the cell membrane, a primary function of which is semipermeability - the ability to allow water to pass through to maintain osmotic balance with its surroundings, but provide a barrier to the passage of almost everything else. When the concentration of solutes around a cell increases, a cell responds by expelling water to re-establish osmotic balance. Loss of semipermeability - when a cell loses the ability to respond to osmotic changes - is a primary cause of damage in cells which have been dehydrated or frozen $[8,17]$, and has been correlated with transitions of membrane lipids to non-bilayer phases, which are no longer capable of keeping the inside and outside of the cell separated.

Despite their potential importance, inverse ribbon phases have previously been studied in only a small number of very low hydration systems of soaps eg [2] and some phospholipids [3, 18-23]. The data that does exist on this phase is limited in phospholipid systems, partly due to poor resolution of higher order Bragg reflections. At present there is no systematic study of the temperature dependence of the structural parameters, or the kinetics of the transition from the $H_{I I}$ phase.

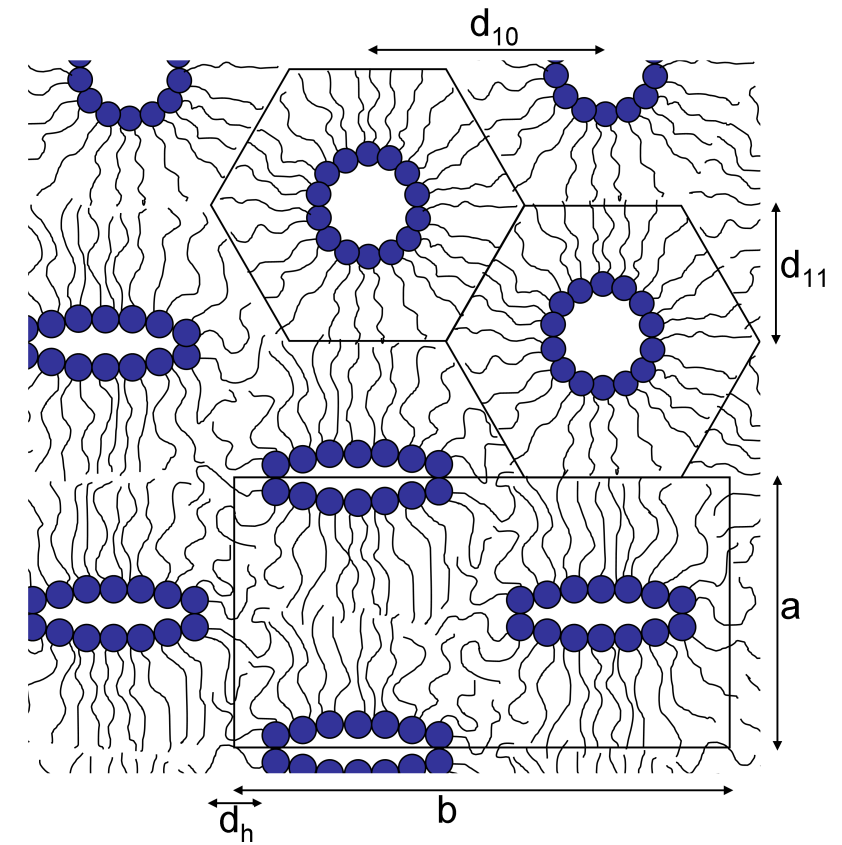

Figure 1: (Color online) Schematic of the $H_{I I}$ and $P_{\alpha}$ phases. The structural parameters of the unit cells for both phases are shown. In the ribbon phase the headgroups do not extend to the end of the ribbons, but rather that the space between ribbons is filled with hydrocarbon, represented by the hydrocarbon distance $d_{h}$.

In this paper we use synchrotron small and wide angle x-ray scattering (SAXS/WAXS) to study the phase behaviour of a low hydration mixture of Dioleoylphosphatidylcholine (DOPC) and Dioleoylphosphatidylethanolamine (DOPE), representative of the most widely occurring phospholipids in nature. We observe an inverse fluid ribbon phase $\left(P_{\alpha}\right)$ which is stable over a wider range of temperatures, and at higher hydrations, than has previously been observed. The system undergoes a transition from $P_{\alpha}$ to $H_{I I}$ over the range 
40-45 ${ }^{\circ} \mathrm{C}$. Upon cooling, the $P_{\alpha}$ phase transforms into the lamellar gel $\left(L_{\beta}\right)$ phase just below $0{ }^{\circ} \mathrm{C}$, without an intermediate fluid lamellar $\left(L_{\alpha}\right)$ phase. We characterize the structural changes occuring during the $H_{I I}$ to $P_{\alpha}$ transition, and determine the temperature dependence of the structural parameters of the $P_{\alpha}$ phase over a wide temperature range. The high flux of 3rd generation synchrotrons allows for short acquisition times with high signal to noise, enabling studies of the kinetics of the transition, with some hysteresis observed.

DOPC and DOPE were obtained from Avanti Polar lipids and used without further purification. The lipids were measured by weight to give the molar ratio DOPC:DOPE 2:1. Samples were hydrated with excess water and vortex mixed while cycling over a range of temperatures to ensure sample homogeneity. Once mixed, the samples were equilibrated over saturated salts at a relative humidity $(\mathrm{RH})$ of $32 \%$ for two weeks at room temperature. Water content of the sample (4.3 wt\%, or 1.9 water molecules per lipid) was determined gravimetrically following experiments by drying samples in DSC pans with pin holes over $P_{2} \mathrm{O}_{5}$ in a vacuum oven at $80^{\circ} \mathrm{C}$.

Synchrotron SAXS/WAXS experiments were carried out on the ChemMatCARS 15ID-D beamline at the Advanced Photon Source (APS), Argonne National Laboratory. Diffraction patterns were recorded on a Bruker 6000 CCD detector over the q-range 0.046 to $1.7 \AA^{-1}$, with the lower q-range (SAXS) probing the primary repeats of the inter water core distance for the $H_{I I}$ phase and the bilayer repeat spacing for the lamellar and ribbon phases. The higher q-range (WAXS) probes the intra-lipid spacing which indicates lipid fluidity. Samples were placed in $1.5 \mathrm{~mm}$ quartz X-ray capillaries (Wolfgang Muller Glas Technik, Berlin) and sealed using Araldite epoxy resin. Temperature control was achieved using a circulating water chiller/heater. Equilibrium measurements (in the range $20{ }^{\circ} \mathrm{C}-80{ }^{\circ} \mathrm{C}$ ) were made after allowing the sample temperature to equilibrate for at least $5 \mathrm{~min}$. Kinetic studies were carried out by equilibrating at $20{ }^{\circ} \mathrm{C}$ then temperature jumping to $80{ }^{\circ} \mathrm{C}$ (rate $\sim 20{ }^{\circ} \mathrm{C} / \mathrm{min}$.), equilibrating for 60 seconds, then temperature jumping back down to $20{ }^{\circ} \mathrm{C}$. Exposures were made at 10 second intervals, and all exposures were 1.2 seconds in duration.

Further equilibrium SAXS measurements below $20{ }^{\circ} \mathrm{C}$ were carried out on a Nanostar (Bruker, Germany) covering the q-range 0.035 to $0.85 \AA^{-1}$. Exposures were of 1 hour duration after equilibrating the sample for $30 \mathrm{~min}$. at the measurement temperature (acquisition times are three orders of magnitude longer than the synchrotron experiments). Samples were mounted in Aluminium sample holders with windows of clear adhesive tape (3M). This instrument is not capable of measuring in the WAXS region, so Differential Scanning Calorimetry (DSC) measurements (Pyris 7 DSC with intracooler (Perkin-Elmer)) were made in order to aid in phase identification. Sam-

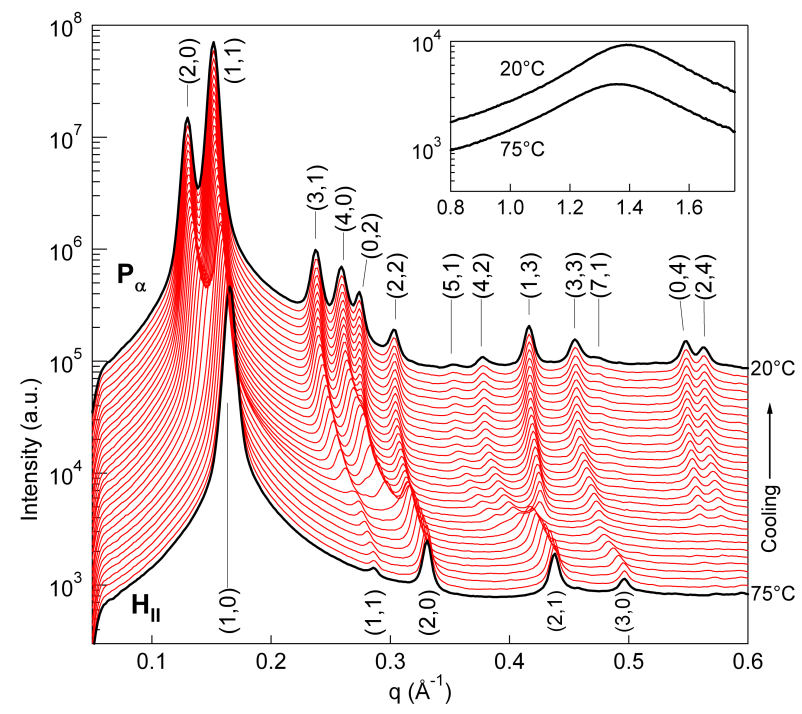

Figure 2: (Color online) SAXS scans during cooling from 80 ${ }^{\circ} \mathrm{C}$ (front) to $20{ }^{\circ} \mathrm{C}$ (back). Scans are displaced vertically for clarity. The scans were taken at $10 \mathrm{~s}$ intervals and are of $1.2 \mathrm{~s}$ duration. Cooling rate was approximately $20{ }^{\circ} \mathrm{C} / \mathrm{min}$. The inset shows the broad wide angle peak, indicative of fluid chains, which does not change significantly over this temperature range.

ples were scanned at $10{ }^{\circ} \mathrm{C} / \mathrm{min}$. and $20{ }^{\circ} \mathrm{C} / \mathrm{min}$., during both heating and cooling.

Fig. 2 shows synchrotron SAXS patterns taken during cooling from $80{ }^{\circ} \mathrm{C}$ to $20{ }^{\circ} \mathrm{C}$ at $\sim 20{ }^{\circ} \mathrm{C} / \mathrm{min}$. Phases were assigned based on the characteristic spacing of higher order reflections. At $75{ }^{\circ} \mathrm{C}, 5$ small angle reflections are evident, with the reflections indexing according to the inverse hexagonal phase $H_{I I}: q_{h k}=\sqrt{\left(h^{2}+h k+k^{2}\right)}$. The broad WAXS reflection centred at $1.36 \AA^{-1}$ (inset) gives the average intra-lipid spacing (ie the spacing between headgroups). The broadness and absence of distinct sharp peaks in this q region indicates the hydrocarbon chains of the lipid molecules have the high degree of disorder characteristic of a fluid phase.

As the sample is cooled, it undergoes a transition over the range $40-45^{\circ} \mathrm{C}$. At the lowest temperature of $20{ }^{\circ} \mathrm{C}$, 12 major reflections are visible. These reflections index according to a rectangular $2 \mathrm{D}$ monoclinic lattice with a systematic absence of $(h, k)$ peaks, $h+k=2 n+1$ indicating centred symmetry: $q_{h k}=2 \pi \sqrt{\left(\frac{h}{a}\right)^{2}+\left(\frac{k}{b}\right)^{2}}$ where the parameters $\mathrm{a}$ and $\mathrm{b}$ denote the short and long unit cell axes respectively, as shown in Fig. 1. This phase is identified as the inverse fluid ribbon phase, $P_{\alpha}$, which consists of ribbon like strips of alternating layers of lipid bilayer and anti-bilayer with disordered fluid hydrocarbon chains ( $\alpha$ conformation). The $P_{\alpha}$ phase has previously been observed in dehydrated DOPE systems at water contents below $0.6 \%$ [18, 20-22]. An inverse ribbon phase with ordered hydrocarbon chains $\left(P_{\delta}\right)$ has also been observed 
in dehydrated DOPE $[19,23]$ as well as in PC systems [3]. To the knowledge of the authors, all observations of the ribbon phase in phospholipid systems have been in anhydrous or dehydrated systems with water contents at or below $0.6 \%$, and most of the previous work has been limited by low resolution of the higher order reflections. The detailed nature of the $H_{I I}-P_{\alpha}$ transition has not previously been systematically studied.

Fig. 3 shows the structural parameters of both phases as functions of temperature. Both kinetic data (Fig. 2) and equilibrium data are shown. Clearly, the temperature has a greater effect on the structure on the $P_{\alpha}$ phase than the $H_{I I}$ phase, with the $H_{I I}$ unit cell dimensions remaining essentially constant over the measured temperature range. On cooling to the $H_{I I}-P_{\alpha}$ transition, there is an immediate transition into the $P_{\alpha}$ phase with the transition complete within two exposures during scanning ( $<20$ seconds). The $P_{\alpha}$ short axis, a, is initially equal to twice the $H_{I I}(1,1)$ planar distance, and remains essentially constant. The long axis, $b$, is equal to twice the $H_{I I}(1,0)$ planar distance at the transition (Fig. 1). Both the agreement in the structural parameters and the speed of the transition demonstrate that the $H_{I I}-P_{\alpha}$ transition involves a distortion of the hexagonal packing of the headgroups into an elongated ribbon, which does not incur a large energy cost. Indeed, DSC measurements show no measurable peak at this temperature. These findings are consistent with the idea [21] that the transition is entropy rather than enthalpy driven.

However, upon cooling there is an extension of the length of the ribbon, with the cell length $b$ following an approximately linear trend as a function of temperature. Both kinetic heating and cooling show the same trends, with some hysteresis, and the equilibration measurements bisect the hysteresis loop. Below $30{ }^{\circ} \mathrm{C}$ the ratio $\frac{b}{a}>2$, allowing the $(4,0)$ and $(0,2)$ reflections of the $P_{\alpha}$ phase to be differentiated in the SAXS data.

The intra-lipid spacing (inset, Fig. 2) decreases from $4.6 \AA$ to $4.5 \AA$ in a roughly linearly fashion as the temperature is reduced, while the FWHM of the intra-lipid WAXS peak remains constant. There is no qualitative change in this peak, indicating there are no gel or crystalline phases in the system. This is in contrast to other reports which have reported an inverse ribbon phase in dehydrated lipid systems with the hydrocarbon chains in the $\delta$ conformation - stiff chains with rotational disorder in a two-dimensional square lattice arrangement.

At lower temperatures $\left(<20^{\circ} \mathrm{C}\right.$ - equilibrium measurements), there is a transition to a lamellar phase at $-4{ }^{\circ} \mathrm{C}$, identified by a change in the spacing of the bragg reflections to the ratios $1: 2: 3: 4$ (data not shown). intralipid (WAXS) data is not available on this instrument, however, DSC heating scans showed a phase transition beginning at $-2.4{ }^{\circ} \mathrm{C}$, with an enthalpy of $27.5 \mathrm{~kJ} / \mathrm{mol}$, consistent with a transition from fluid to ordered chains, such as the $L_{\alpha}$ to $L_{\beta}$ (fluid to gel) transition. In ad-

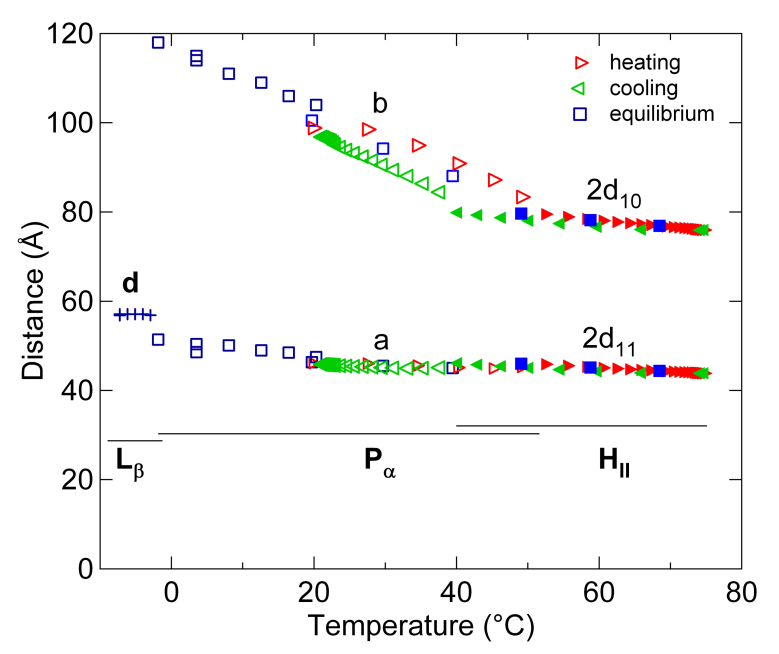

Figure 3: (Color online) Structural dimensions as a function of temperature. Filled symbols are the $H_{I I}$ phase, open symbols are the $P_{\alpha}$ phase. The temperature ranges over which the three phases are identified are indicated by horizontal lines. Kinetic runs are indicated by right and left pointing triangles for heating and cooling respectively. Squares are equilibrium measurements (both heating and cooling). Plusses indicate the lamellar gel phase. Measurements at temperatures $\leq 20$ ${ }^{\circ} \mathrm{C}$ were carried out on a lab based instrument, the others are synchrotron measurements.

dition, the measured bilayer thickness is $10 \%$ larger in the lamellar phase than in the $P_{\alpha}$ phase, again indicative of a fluid-gel transition. The slight offset between the transition temperatures is due to the fact that the SAXS data is measured at equilibrium, whereas the DSC measuremtns are made during scanning at $10{ }^{\circ} \mathrm{C} / \mathrm{min}$.

Interestingly, there is no discontinuity in the cell area as a function of temperature over the phase transition with the ribbon unit cell area equal to twice the $H_{I I}$ unit cell area at the transition. As this is the case, one would assume that at the $H_{I I}-P_{\alpha}$ transition, the number of lipids around the $H_{I I}$ water core and the number of lipids in half a ribbon should be the same.

The number of lipids around the $H_{I I}$ core can be calculated from the water core radius and the repeat spacing. Using literature values for the partial specific volumes of DOPC [24, 25] and DOPE [4], and knowing the mass fractions, the volume fraction of water $\phi_{W}=\frac{V_{W}}{V_{L}+V_{W}}$ can be calculated. The partial specific volumes are assumed not to change appreciably unless there is a chain freezing/melting transition, such as the $L_{\alpha}-L_{\beta}$ transition [26]. The $H_{I I}$ water core radius is then related to the water volume fraction by $R_{W}=2 d_{11} \sqrt{\phi_{W} \frac{\sqrt{3}}{2 \pi}}$ [4]. For the hexagonal phase at $75{ }^{\circ} \mathrm{C}$ this gives $R_{W}=4.9 \AA$. From this, and the $\mathrm{d}$ spacing, one can then calculate the distance from the edge of the water core to the edge of the hexagonal unit cell along the $(1,1)$ and $(1,0)$ axes, i.e. the minimum and maximum lengths of the lipids in 
this system. In this case $18.2 \AA$ and $21.7 \AA$ respectively, with an average value of $\sim 19.9 \AA$.

However, the WAXS peak derives from scattering at some average point along the polar headgroup of the lipid. The polar head group thickness has been estimated as $\sim 8 \AA$ for DOPE at similar hydrations in the $H_{I I}$ phase [27], and $10 \AA$ for phosphatidylcholines in the lamellar phase [28]. Therefore if we assume that the intra-lipid WAXS peak corresponds to halfway along the polar headgroup, this adds $\sim 5 \AA$ to the $4.8 \AA$ water core radius, giving a circumference of $\sim 61 \AA$. The intra-lipid $\mathrm{d}$ spacing at the transition is $4.54 \AA$, meaning $\sim 14$ lipids could fit around the circumference of the water core.

The number of lipids per ribbon unit cell can be determined by assuming that the horizontal hydrocarbon distance between the ribbons $\left(d_{h}\right.$ in Fig. 1$)$ can be approximated as the average lipid length in the $H_{I I}$ phase $(19.9 \AA)$ minus the headgroup length $(9 \AA)$. Using the the unit cell length ( $b=84.6 \AA$ at the transition) and the intra-lipid spacing $(4.54 \AA)$, leads to an estimate of $\sim 16$ lipids per ribbon, which is consistent, within the assumptions, with the number of lipids around the $H_{I I}$ core. Future electron density studies might shed further light onto the nature of this transition.

In this paper we have demonstrated for the first time a $P_{\alpha}$ phase stable over a wide temperature range, with a direct transition between the $P_{\alpha}$ phase and gel lamellar phase (with no intermediate lamellar fluid phase). In other words, the $P_{\alpha}$ phase exists as an intermediate phase between the gel lamellar and $H_{I I}$ phases. Furthermore we have shown that the $P_{\alpha}$ phase can exist at a higher water content in mixed DOPC/DOPE mixtures than in single component systems of either constituent. Of course biological membranes are much more complicated, with many different lipid species, as well as proteins and other components. However, as previous work has shown, differential hydration of different membranes components phase can lead to phase separations [11, 12, 29], concentrating lipids with tendencies to form inverse phases. Similar effects are expected to occur as membranes are brought into close proximity during fusion.

The results presented here are important for our understanding of freezing and dehydration damage in biological membranes, as well as being potentially relevant to membrane fusion. The fact that this relatively simple mixture exhibits a stable $P_{\alpha}$ phase over such a wide temperature range is an indication that even simple two component model systems behave in ways very different from the single component lipid systems which have been most widely studied to date. This highlights the importance of studying more complex model membrane systems, in order to better understand biological membranes.

The authors thank Bob Shanks for the use of the DSC. The use of the ChemMatCARS sector was supported by the Australian Synchrotron Research Program, funded by the Commonwealth of Australia under the Major National Research Facilities Program. Use of the Advanced Photon Source was supported by the U. S. Department of Energy, Office of Science, Office of Basic Energy Sciences, under Contract No. DE-AC02-06CH11357. Kent is supported by an AINSE postgraduate award.

* Electronic address: gary.bryant@rmit.edu.au

[1] V. Luzzati and F. Husson, J. Cell Biol. 12, 207 (1962).

[2] V. Luzzati, in Biological Membranes: Physical Fact and Function, edited by D. Chapman (Academic Press, London, 1968), vol. 1, pp. 71-123.

[3] A. Tardieu, V. Luzzati, and F. C. Reman, J. Mol. Biol. 75, 711 (1973).

[4] M. W. Tate and S. M. Gruner, Biochem. 28, 4245 (1989).

[5] M. W. Tate, E. Shyamsunder, S. M. Gruner, and K. L. D'Amico, Biochem. 31, 1081 (1992).

[6] R. M. Epand, Chem. Phys. Lipids 81, 101 (1996).

[7] S. M. Gruner, Science 297, 1817 (2002).

[8] L. M. Crowe and J. H. Crowe, Arch. Biochem. Biophys. 217, 582 (1982).

[9] J. H. Crowe, L. M. Crowe, and R. Mouradian, Cryobiol. 20, 346 (1983).

[10] M. Caffrey, Biochem. 24, 4826 (1985).

[11] G. Bryant, J. M. Pope, and J. Wolfe, Eur. Biophys. J. 21, 223 (1992).

[12] G. Bryant and J. Wolfe, Cryo-Letters 13, 23 (1992).

[13] J. H. Crowe and L. M. Crowe, Liposome Technology (CRC Press, Boca Raton, Fl., 1993), vol. 1, pp. 229-252.

[14] L. Yang and H. W. Huang, Science 297, 1877 (2002).

[15] L. Yang and H. W. Huang, Biophys. J. 84, 1808 (2003).

[16] L. Yang, L. Ding, and H. W. Huang, Biochem. 42, 6631 (2003).

[17] W. J. Gordon-Kamm and P. L. Steponkus, Proc. Natl. Acad. Sci. USA 81, 6373 (1984).

[18] W. Pohle and C. Selle, Chem. Phys. Lipids 82, 191 (1996).

[19] E. Y. Shalaev and P. L. Steponkus, Biochim. Biophys. Acta 1419, 229 (1999).

[20] H. Binder and W. Pohle, J. Phys. Chem. B 104, 12039 (2000).

[21] H. Binder, B. Kohlstrunk, and W. Pohle, J. Phys. Chem. B 104, 12049 (2000).

[22] W. Pohle, C. Selle, D. R. Gauger, and K. Brandenburg, J. Biomol. Struct. Dyn. 19, 351 (2001).

[23] E. Y. Shalaev and P. L. Steponkus, Biochim. Biophys. Acta 1514, 100 (2001).

[24] S. Tristram-Nagle, H. I. Petrache, and J. F. Nagle, Biophys. J. 75, 917 (1998).

[25] B. W. Koenig and K. Gawrisch, Biochim. Biophys. Acta 1715, 65 (2005).

[26] J. F. Nagle and D. A. Wilkinson, Biophys. J. 23, 159 (1978).

[27] R. P. Rand and N. L. Fuller, Biophys. J. 66, 2127 (1994).

[28] T. J. McIntosh and S. A. Simon, Biochem. 25, 4948 (1986).

[29] G. Bryant and J. Wolfe, Eur. Biophys. J. 16, 369 (1989). 